\title{
The Posterior Lateral Femoral Contusion Sign (Aamer Botchu Sign): an Ancillary Sign of MCL Injury
}

\author{
A. Iqbal', E. McLoughlin11, D. Beale², SL. James'1, R. Botchu \\ 1 Department of Musculoskeletal Imaging, Royal Orthopaedic Hospital, Birmingham, UK \\ 2 Heath lodge clinic, Solihull, UK
}

\section{CORRESPONDING AUTHOR:}

Rajesh Botchu

Department of Musculoskeletal Radiology

The Royal Orthopedic Hospital

Bristol Road South

Northfield, Birmingham, UK

Phone: 00441216854000

E-mail: drbrajesh@yahoo.com

DOI:

10.32098/mltj.01.2020.16

LEVEL OF EVIDENCE: 4

\begin{abstract}
SUMMARY
Medial collateral ligament complex (MCL) injury is the commonest injury in the knee. Assessment of the osseous edema pattern can help predict the mechanism of a soft tissue injury.

Material and methods. We performed a retrospective study to analyse the osseous edema involving the lateral femoral condyle and associated soft tissue injuries.

Results. Of the 334 patients with radiological diagnosis of MCL sprain, there were 53 patients with a clinical history of valgus stress injury and bone bruising of the lateral femoral condyle. These patients were divided into two cohorts depending in the presence of isolated MCL injury (cohort one) or co-existing MCL and ACL injuries (cohort two). There were 23 patients in cohort one; mean age was 38 years (range 16-68) with 15 males and 8 females. In cohort two, there were 30 patients; mean age was 30.5 years (range 14-62) with 20 males and 10 females. Assessment of the location of bone bruising within the lateral femoral condyle revealed bone bruising involving the posterior third in 19/23 cases in cohort one compared to $1 / 30$ patients in cohort 2 which was statistically significant $(\mathrm{p}<0.001)$. These were high grade 2 or grade 3 sprain involving the superficial and deep components of the MCL, posterior oblique ligament and medial patellofemoral retinaculum.

Conclusion. Based on our results with conclude that isolated osseous edema of the posterior third of the lateral femoral condyle is associated with isolated MCL injury. (Aamer Botchu sign)
\end{abstract}

\section{KEY WORDS}

Posterior; lateral; femoral; condyle; edema

\section{INTRODUCTION}

Medial collateral ligament (MCL) injury is the commonest ligamentous injury to occur in the knee. It most commonly occurs following valgus force in sports related injuries, motor vehicle accidents or falling from a height (1). The MCL maybe injured in isolation or in conjunction with other structures such as the anterior cruciate ligament (ACL) and the medial meniscus. Magnetic resonance imaging (MRI) is the investigation of choice for evaluating the soft tissue and osseous abnormalities of the knee following trauma. In particular, the osseous injuries are often occulted on radiographs. Following injury, bone bruising (osseous oedema) is a common finding on MRI manifesting as decreased signal intensity on $\mathrm{T} 1$ weighted sequences and increased signal on fluid sensitive sequences. The appearances are thought to represent areas of haemorrhage, oedema or hyperaemia secondary to trabecular injury caused by the different forces acting on the knee such as impact from a direct blow to the bone, compressive forces of adjacent bones during impaction or traction forces of soft tissue structures during avulsion injury $(2,3,4)$. Assessment of the distribution of the pattern of the bone bruising allows a prediction of the associated soft tissue injuries that maybe present. A number of injury patterns have been described which include: clip injury, pivot shift injury, dashboard injury, hyperextension injury and lateral patella dislocation (figure 1)

Following a lateral clip injury (valgus stress), the medial collateral ligament complex maybe injured in isolation or in conjunction with the ACL and menisci depending on the degree of knee flexion. The aim of this study is to differentiate the bone marrow oedema pattern on the lateral aspect of the lateral femoral condyle following a clip injury and 


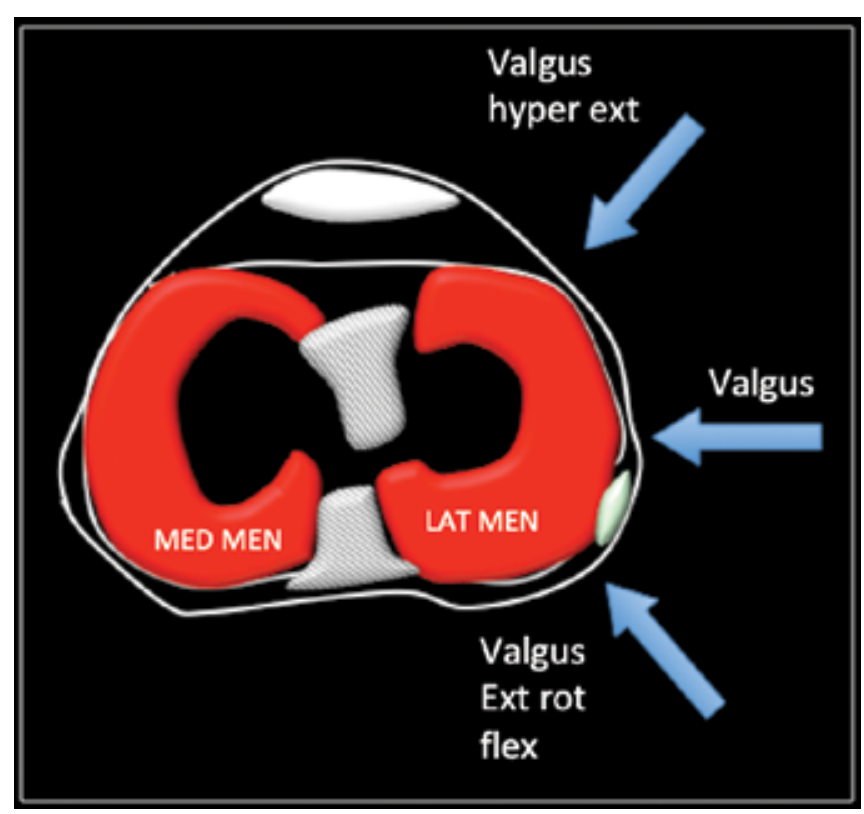

Figure 1. Axial animation showing angle of force in valgus hyperextension (valgus hyper ext), valgus and valgus external rotation and flexion (valgus ext rot flex).

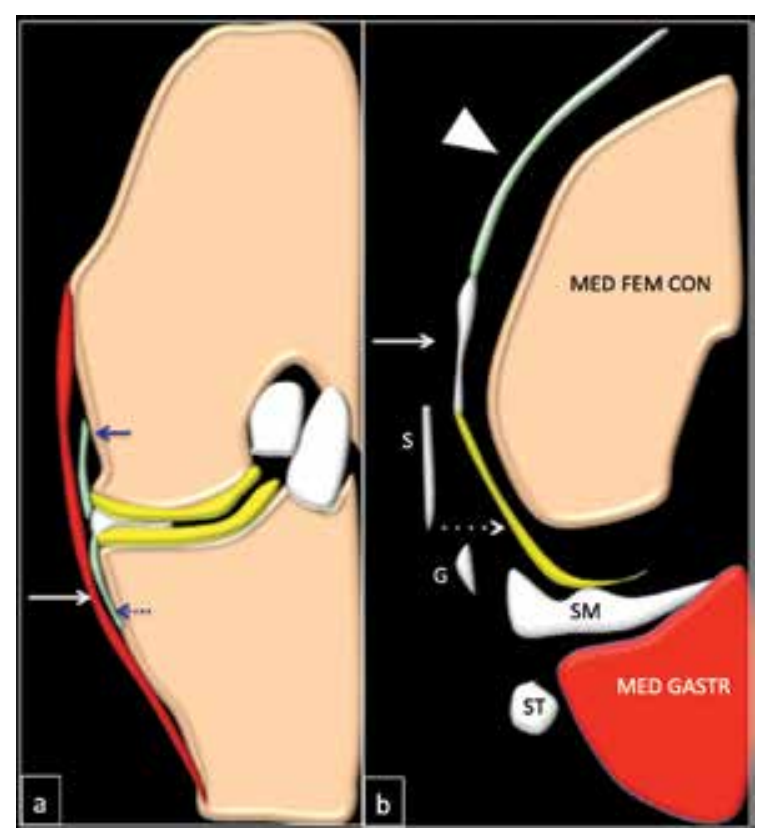

Figure 2. Animation of coronal and axial section of medial knee showing normal anatomy, white arrow (superficial $\mathrm{MCL}$ ), black arrow (meniscofemoral ligament), dashed black arrow (meniscotibial ligament) arrow head (patellofemoral retinaculum), dashed white arrow (medial oblique ligament). G (gracilis), SM (semimembranosus), ST (semitendinosus), S(sartorius), Med gastr (medial gastrocnemius), Med fem con (medial femoral condyle). to predict the pattern of resulting soft tissue injury such as isolated MCL or coexisting ACL and MCL injury.

\section{ANATOMY}

The two important ligaments which stabilize the medial aspect of the knee are the MCL and the posterior oblique ligament (POL). The MCL has a superficial mcl (sMCL- tibial collateral ligament) and deep mcl (dMCL- meniscofemoral and meniscotibial ligaments) component. The sMCL is the largest structure on the medial side of the knee joint. It has a single point femoral insertion on the medial epicondyle and a dual point distal tibial insertion (5). The proximal aspect of the dual point insertion inserts onto soft tissue closely related to the anterior aspect of the semimembranosus tendon and the distal aspect of the dual point insertion is a bony insertion onto the medial tibia close to the pes anserinus (figure 2). The soft tissue insertion of the sMCL is the main stabilizer against valgus stress during flexion whilst the distal bony insertion is responsible for external rotation from 30-60 degrees of knee flexion and internal rotation.

\section{METHODS}

Following local committee ethical approval, a retrospective study of our imaging database was performed to identify all patients with an MRI diagnosis of MCL strain between 2014 and 2019 (6). The distribution of bone bruising in the lateral femoral condyle was assessed and compared in patients with injury to the medial supporting structures in isolation (MCL, MPFL (medial patellofemoral ligament), POL) with patients who had co-existing medial supporting structure injury and ACL injury. In order to assess the location of bone marrow oedema, the lateral femoral condyle was divided into anterior, middle and posterior thirds. This was along the level of the anterior horn, body and posterior horns of the lateral meniscus. Patients with a history of previous surgery or an absence of bone bruising on MRI were excluded. The ACL was assessed for complete or partial thickness tears. The medial supporting structures (superficial and deep MCL, medial patella-femoral ligament (MPFL) posterior oblique ligament (POL)) were assessed and the degree of sprain was documented. The images were reviewed by a Consultant Musculoskeletal Radiologist and Musculoskeletal fellow. The data was analysed using chi square test and $\mathrm{p}$ values of less than 0.05 were considered to be significant.

\section{RESULTS}

Our initial search revealed 334 patients who had a radiological diagnosis of MCL sprain. Of these, there were 53 


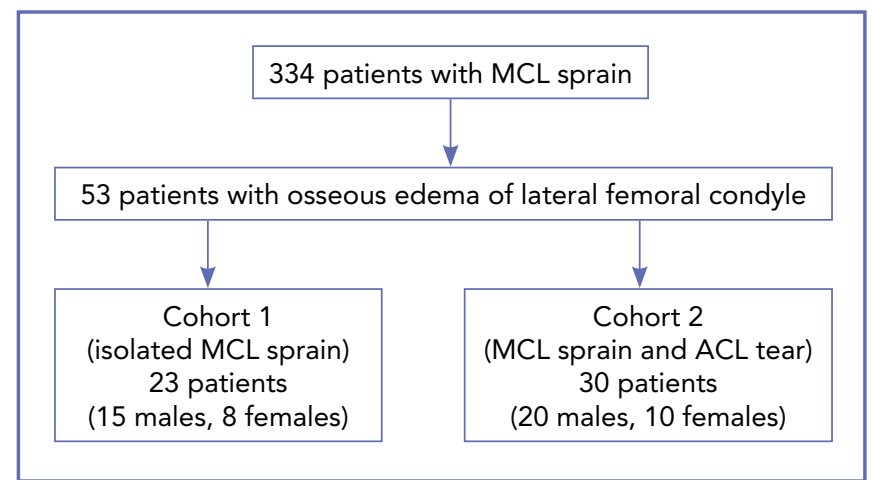

Figure 3. Image showing cohorts of patients in the study.

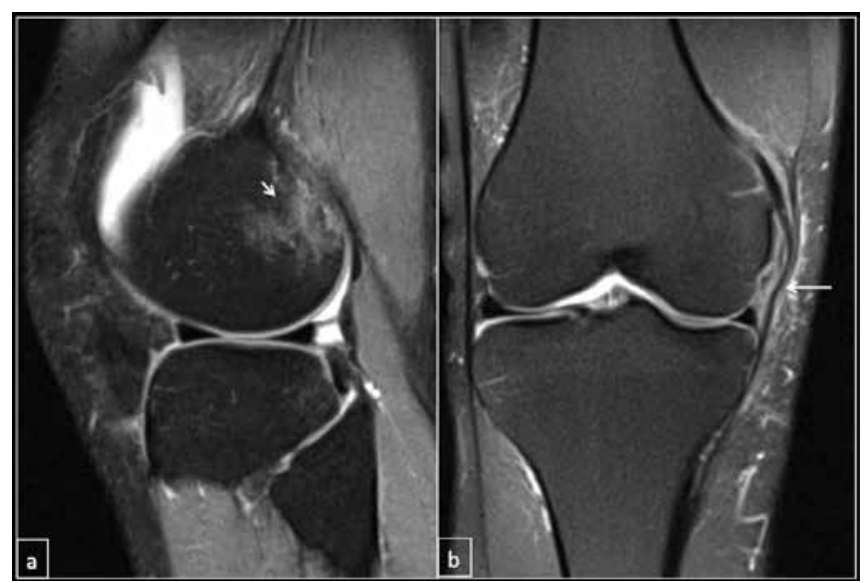

Figure 4. Proton density fat suppressed (PDFS) sagittal (a) and coronal (b) showing marked osseous edema involving the posterior third of the lateral femoral condyle (small arrow) with grade 2 sprain of the femoral attachment of the superficial component of MCL and meniscofemoral ligament (arrow).

Table I. Pattern of injuries with osseous edema of posterior third of the lateral femoral condyle.

\begin{tabular}{lll}
\hline Injury & yes & no \\
\hline MCL & 19 & 4 \\
\hline ACL & 1 & 29 \\
\hline & p value & $<0.0001$ \\
\hline
\end{tabular}

Table II. Pattern of injuries with osseous edema of middle third of the lateral femoral condyle.

\begin{tabular}{lll}
\hline Injury & yes & no \\
\hline MCL & 9 & 14 \\
\hline ACL & 19 & 11 \\
\hline & p value & 0.08 \\
\hline
\end{tabular}

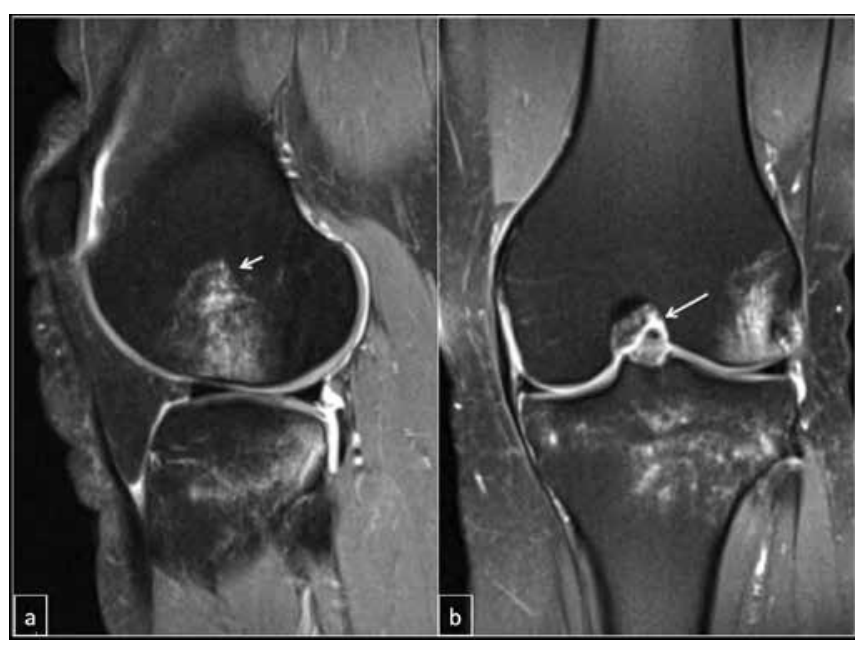

Figure 5. Proton density fat suppressed (PDFS) sagittal (a) and coronal (b) showing marked osseous edema involving the middle third part of the lateral femoral condyle (small arrow), osseous edema of the proximal tibia (pivot shift pattern of osseous edema) with full thickness tear of the ACL (arrow).

patients with a clinical history of valgus stress injury and bone bruising of the lateral femoral condyle. These patients were divided into two cohorts depending in the presence of isolated MCL injury (cohort one) or co-existing MCL and ACL injuries (cohort two). There were 23 patients in cohort one, mean age was 38 years (range 16-68) with 15 males and 8 females. In cohort two, there were 30 patients, mean age was 30.5 years (range 14-62) with 20 males and 10 females (figure 3).

Assessment of the location of bone bruising within the lateral femoral condyle revealed bone bruising involving the posterior third in 19/23 cases in cohort one compared to $1 / 30$ patients in cohort 2 which was statistically significant $(\mathrm{p}<0.001)$ (table I) (figure 4). In the middle third of the femoral condyle, bone bruising was identified in $9 / 23$ patients in cohort one compared to $19 / 30$ in cohort 2 with no statistical significance $(\mathrm{p}=0.08)$ (table II) (figure 5). In the anterior third of the lateral femoral condyle, 6/23 patients in cohort one demonstrated bone bruising whereas 26/30 patients demonstrated this pattern in cohort two which was statistically significant $(\mathrm{p}<0.0001)$ (table III). The average area of bone bruise in lateral femoral condyle in cohort 1 was $632 \mathrm{~mm}^{2}$ (range of 1152-169 $\mathrm{mm}^{2}$ ). There was no correlation between the area of osseous edema and grade of sprain of MCL. (average bone bruise in grade 1 sprain $462 \mathrm{~mm}^{2}$, grade 2 sprain was $668 \mathrm{~mm}^{2}$, grade 3 sprain was $619 \mathrm{~mm}^{2}$ ). There were three cases of medial meniscal tear in cohort 1 . 
Table III. Pattern of injuries with osseous edema of anterior third of the lateral femoral condyle.

\begin{tabular}{lll}
\hline Injury & yes & no \\
\hline MCL & 6 & 17 \\
\hline ACL & 26 & 4 \\
\hline & p value & $<0.0001$ \\
\hline
\end{tabular}

\section{DISCUSSION}

Bone bruising around the knee is commonly encountered on knee MRI. In the context of trauma, the distribution of bone bruising is the residual footprint from the injury. Understanding the various patterns of bone bruising helps one to predict the mechanism of injury and thus assess for associated soft tissue injuries that occur with specific injury patterns. There are five classic bone bruising patterns around the knee which include: pivot shift injury, dashboard injury, hyperextension injury, clip injury and lateral patella dislocation (7). Of these injuries, clip injury and dashboard injury are related to contact with an external force whilst the remaining three injury patterns are related to non-contact injury.

The clip injury is common in american football players and occurs when a pure valgus stress is applied to a partially flexed knee (10-30 degrees) (8). The bone bruising is most prominent on the lateral femoral condyle secondary to the direct blow whilst a smaller area of marrow oedema maybe visualized on the medial femoral condyle resulting from avulsive stress from the MCL. Varying degrees of MCL injury is the commonest soft tissue abnormality associated with this traumatic and particular mechanism of injury (9). The femoral portion of the MCL fibres is most frequently injured. MCL sprains are divided into three grades. Grade 1 sprain on MR images is visualized as oedema superficial (medial) to the ligament without disruption of fibres. Grade 2 sprain is oedema superficial to the ligament and oedema partially extending through the ligament with partial disruption of fibres. Grade 3 sprain is when there is complete discontinuity of fibres (10). In 1950, O'Donoghue described the 'unhappy triad' as a severe variant of the classic valgus clip injury, which occurs when there is increasing degrees of knee flexion with the foot fixed to the ground. This results in a lateral rotation of the leg and over abduction resulting in coexisting injuries to the ACL, MCL and medial meniscus (11). A further study by Shelbourne and co-authors in 1991 found that injuries to the lateral meniscus occur more frequently than the medial meniscus with this mechanism of injury and that medial meniscus injuries never occur in the absence of lateral meniscal tears (12).

Whilst there have been numerous articles describing the various bone bruising and soft tissue injury patterns but there is no article focussing on the location of bone bruising in the lateral femoral condyle following a valgus stress (clip) injury $(12,13,14)$.

Our study has shown that there is statistically significant correlation between the location of bone bruising and the presence of isolated MCL or co-existing MCL and ACL injuries. We have found that when the bone bruising is located on the posterior third of the lateral femoral condyle, injury to the MCL and surrounding medial supporting structures such as MPFL and POL occur with an intact ACL $(\mathrm{P}<0.001)$. Whereas, if the bone bruising involves the anterior third of the femoral condyle then injury to the ACL and MCL along with medial supporting structures occurs $(\mathrm{P}<0.001)$. We describe this pattern of bone bruising involving the posterior third of the lateral femoral condyle as the $\mathrm{AB}$ (Aamer Botchu) sign.

Our study has some limitations. One is this being a retrospective study. The other being the relatively low number of cases but this could be due to the fact that most injuries are managed symptomatically either with rest, analgesics or physiotherapy before obtaining MRI by which time the osseous edema might have resolved.

\section{CONCLUSIONS}

Valgus stress injuries of the knee are common and result in various injury patterns ranging from isolated MCL injury to severe injuries such as Donoghues unhappy triad, which includes injury to the ACL, MCL and medial meniscus. Our study has shown that the $\mathrm{AB}$ sign is associated with isolated MCL injury.

\section{CONFLICT OF INTERESTS}

The authors declare that they have no conflict of interests

\section{REFERENCES}

1. Indelicato PA. Isolated medial collateral ligament injuries in the knee. J Am Acad Orthop Surg. 1995; 3(1):9-14.

2. Steiner RM, Mitchell DG, Rao VM, Schweitzer ME. Magnetic resonance imaging of diffuse bone marrow disease. Radiol Clin North Am 1993; 31:383-409.

3. Kapelov SR, Teresi LM, Bradley WG Bone contusion of the knee: increased lesion detection with fast spin-echo MR imaging with spectroscopic fat saturation. Radiology 1993; 189:901- 904.

4. Lynch TC, Crues JV III, Morgan FW. Bone abnormalities of the knee: prevalence and significance at MR imaging. Radiology 1989; 171:761-766.

5. Prade RF, Engebretsen AH, Ly TV, Johansen S, Wentorf FA, Engebretsen L. The anatomy of the medial part of the knee. J Bone Joint Surg Am 2007;89:2000-2010. 
6. Padulo J, Oliva F, Frizziero A, Maffulli N. Muscles, Ligaments and Tendons Journal - Basic principles and recommendations in clinical and field Science Research: 2018 update. MLTJ 2018; 8(3): $305-307$.

7. Sanders TG, Medynski MA, Feller JF, Lawhorn KW. Bone Contusion Patterns of the Knee at MR Imaging: Footprint of the Mechanism of Injury. RadioGraphics 2000; 20:S135-S151

8. Derscheid GL, Garrick JG. Medial collateral ligament injuries in football: non-operative management of grade I and grade II sprains. Am J Sports Med 1981; 9:365-368.

9. Fetto JF, Marshall JL. Medial collateral ligament injuries of the knee: a rationale for treatment. Clin Orthop 1978; 132:206-218.

10. Rasenberg EIJ, Lemmens JAM, van Kampen A, Schoots F, Bloo HJKC, Wagemakers HPA. Grading medial collateral ligament injury: comparison of MR imaging and instrumented valgus-varus laxity test-device. A prospective double-blind patient study. European Journal of Radiology. 1995;21(1):18-24
11. Odonoghue DH. Surgical treatment of fresh injuries to the major ligaments of the knee. J Bone Joint Surg. 1950; 32A: 721-738.

12. Shelbourne Kd, Nitz PA. The O'Donoghue triad revisited Combined knee injuries involving anterior cruciate and medial collateral ligament tears. The American Journal of Sports Medicine. 1991;19 (5): 474-477.

13. Chin YC1, Wijaya R, Chong le R, Chang HC, Lee YH. Bone bruise patterns in knee injuries: where are they found? Eur J Orthop Surg Traumatol. 2014;24(8):1481-7.

14. Yoon KH, Yoo JH, Kim KI. Bone contusion and associated meniscal and medial collateral ligament injury in patients with anterior cruciate ligament rupture.J Bone Joint Surg Am. 2011;17;93(16):1510-8.

15. Pezeshki S, Vogl TJ, Pezeshki MZ, Daghighi MH, Pourisa M. Association of the type of trauma, occurrence of bone bruise, fracture and joint effusion with the injury to the menisci and ligaments in MRI of knee trauma. Muscles Ligaments Tendons J. 2016;19;6(1):161-6. 